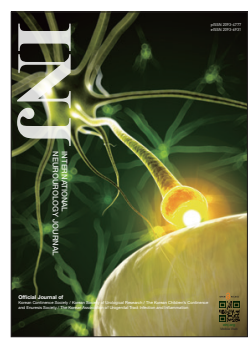

\title{
Molecular and Cellular Substrates of Neuroinflammation in Alzheimer Disease and Depression
}

\author{
Heh-In Im (D) https://orcid.org/0000-0002-4763-5009
}

Convergence Research Center for Diagnosis, Treatment and Care System of Dementia (DTC), Korea Institute of Science and Technology (KIST), Seoul, Korea E-mail: him@kist.re.kr

Alzheimer disease and major depressive disorder are rapidly arising as the major societal concern worldwide. Studies have consistently pointed out that both Alzheimer disease and depression are marked by elevated neuroinflammation from polygenic activation of microglia and astrocytes. In this special issue of the International Neurourology Journal (INJ), we focus on the molecular and cellular origin of neuroinflammation in $\mathrm{Al}$ zheimer disease and depression.

In Alzheimer disease, amyloid- $\beta$ aggregation and tau hyperphosphorylation create amyloid plaques and neurofibrillary tangles, which could subsequently activate pattern recognition receptors on the brain's immune cells including microglia and astrocytes and thereby trigger the release of pro-inflammatory mediators that contribute to Alzheimer disease pathogenesis [1].

In the pathogenesis of Alzheimer disease, the brain-enriched epigenetic regulator MeCP2 (methyl-CpG binding protein 2) can induce glial senescence as well as dysregulate Alzheimer disease risk genes, which consequently accelerate multiple aspects of Alzheimer disease pathology including neuroinflammation [2].

Hypoxia is a major cause of neuroinflammation in the brain [3] and contributes to the pathogenesis of neurodegenerative diseases including Alzheimer disease. The synaptic component neuregulin-1 (NRG1) protects neurons against hypoxic insult [4], which is in line with previous studies demonstrating that NRG1 reduces neuroinflammation in experimental models.
In depression, cryptogenic increase in pro-inflammatory molecules can activate microglia and astrocytes in the brain, which leads to pathological release of pro-inflammatory mediators and potentiate neuroinflammation in a vicious cycle [5].

Neuroinflammation underlies symptoms and pathology in a variety of human diseases and disorders. Hallmarks of Alzheimer disease (amyloid plaques and neurofibrillary tangles), hypoxia and cellular stress, or yet unknown factors in depression can pathologically activate microglia and astrocytes to submerge the brain in pro-inflammatory mediators and give rise to neuroinflammation. From this issue, we hope to provide deeper insight into the causal factors and links between neuroinflammation and major brain diseases. In addition, I am greatly thankful to all the invited authors for the valuable contribution they provided.

- Conflict of Interest: No potential conflict of interest relevant to this article was reported.

\section{REFERENCES}

1. Yang SH. Cellular and molecular mediators of neuroinflammation in Alzheimer disease. Int Neurourol J 2019;23(Suppl 2):S54-62.

2. Kim B, Choi Y, Kim HS, Im HI. Methyl-CpG binding protein 2 in Alzheimer dementia. Int Neurourol J 2019;23(Suppl 2):S72-81.

3. Eltzschig HK, Carmeliet P. Hypoxia and inflammation. N Engl J Med 2014;364:656-65. 
4. Yoo SY, Yoo JY, Kim HB, Baik TK, Lee JH, Woo RS. Neuregulin-1 protects neuronal cells against damage due to CoCl2-Induced hypoxia by suppressing Hypoxia-Inducible Factor-1 $\alpha$ and P53 in
SHSY5Y cells. Int Neurourol J 2019;23(Suppl 2):S111-8.

5. Maeng SH, Hong H. Inflammation as the potential basis in depression. Int Neurourol J 2019;23(Suppl 2):S63-71. 